\title{
BMJ Open Integrated management of type 2 diabetes and gestational diabetes within multi-morbidity conditions in Africa: a systematic review protocol
}

\author{
Jean Claude Mutabazi, ${ }^{1,2}$ Mahmoud M Werfalli, ${ }^{3}$ Angeli Rawat, ${ }^{4}$ Ezekiel Musa,,${ }^{3,5}$ \\ Shane A Norris, ${ }^{5,6}$ Katherine Murphy, ${ }^{3,5}$ Helen Trottier, ${ }^{7,8}$ Naomi Levitt, ${ }^{3,5}$ \\ Christina Zarowsky ${ }^{2,7}$
}

To cite: Mutabazi JC, Werfalli MM, Rawat $\mathrm{A}$, et al. Integrated management of type 2 diabetes and gestational diabetes within multi-morbidity conditions in Africa: a systematic review protocol. BMJ Open 2019;9:e023684. doi:10.1136/ bmjopen-2018-023684

- Prepublication history and additional material for this paper are available online. To view these files, please visit the journal online (http://dx.doi. org/10.1136/bmjopen-2018023684).

Received 25 April 2018 Revised 24 January 2019 Accepted 31 January 2019

Check for updates

(C) Author(s) (or their employer(s)) 2019. Re-use permitted under CC BY-NC. No commercial re-use. See rights and permissions. Published by BMJ.

For numbered affiliations see end of article.

Correspondence to Jean Claude Mutabazi; mutajeanc@yahoo.fr

\section{ABSTRACT}

Introduction Multi-morbidity, defined as the co-existence of more than one chronic condition in one person, has been increasing due to comorbid non-communicable and infectious chronic diseases (CNCICDs). Type 2 diabetes (T2D) and gestational diabetes mellitus (GDM) incidences within the CNCICDs conditions are increasing and overwhelming already weak and under-resourced healthcare systems in Africa. There is then an urgent need for the integrated management of CNCICDs. We aim to review the integrated management of T2D and GDM within multi-morbidity conditions in Africa.

Methods Studies that have assessed the integrated management of T2D and GDM within multi-morbidity conditions in Africa will be considered based on the Population, Intervention, Comparator and Outcome method: population (adult diagnosed with T2D and GDM, who also have other diseases, non-communicable diseases (NCDs) and infectious, in public primary and secondary healthcare facilities in Africa); Intervention (integrated management of T2D and GDM, also suffering from other diseases in Africa), Comparator (Unintegrated management of T2D and GDM in Africa) and Outcomes (integrated management of T2D and GDM in Africa). The following databases Cochrane Library, MEDLINE, PubMed and SCOPUS, the WHO International Clinical Trials Registry Platform, among others will be searched. Two reviewers (JCM and MW) will independently screen, select eligible studies and extract data. Discrepancies will be resolved by consensus or by a discussion with the third author (AR). Quality of included studies will be assessed using both the newly developed tool, 'the Cochrane Collaboration Risk of Bias Tool' and 'Risk Of Bias In Non-randomised Studies - of Interventions (ROBINS-I)". A narrative synthesis of extracted data and meta-analysis, if necessary will be conducted and then reported according to the preferred reporting items for systematic review and meta-analysis.

Ethics consideration and dissemination By only using the published data, there is no ethics approval required for this study. This systematic review will be included in JCM's $\mathrm{PhD}$ thesis and its findings will also be disseminated through peer-reviewed publication and conference presentation.

PROSPERO registration number CRD42016046630.
Strengths and limitations of this study

- Substantial search strategy to identify relevant studies will be adopted, a large number of online databases will be searched, public health websites will be manually searched and credible experts will be consulted.

- Study results will be assessed and reported in accordance with relevant guidelines for quality assessment of systematic reviews.

- Scarcity of eligible studies for selection and inclusion is expected.

- Reviewers will not be blinded during data extraction and quality assessment stages.

\section{INTRODUCTION}

The WHO global report in 2016 estimated that 415-422 million adults worldwide had diabetes in 2014-2015 and that diabetes caused 5 million deaths in 2015, with an estimated US\$673 billion of total global health expenditure in diabetes care. ${ }^{12}$ In the African region, there were an estimated 14.2 million people with diabetes in 2015 increasing to 34.2 million in $2040 .{ }^{13}$ With the expected rural depopulation causing increased exposure to urban environments and diabetogenic lifestyles such as inactivity, obesity, depression, smoking among others, diabetes cases are expected to increase by $54 \%$ to 642 million worldwide by $2040 .^{3-5}$

Globally, the burden of non-communicable diseases (NCDs) is rising. Low- and middle-income countries are most affected by changes in patterns of population age distributions, fertility, life expectancy, morbidity and mortality, known as the 'epidemiological transition'. ${ }^{6}$ In Africa, especially in sub-Saharan Africa, this is occurring against a background of continuing infectious disease epidemics (ie, HIV and tuberculosis), increasingly 
becoming a coinfection epidemic that requires an integrated response. ${ }^{7}$ Consequently, multi-morbidity defined as the co-existence of more than one chronic condition in one person, has been increasing due to comorbid non-communicable and infectious chronic diseases (CNCICDs) ${ }^{8}$ Given the risk factors and complex care needs of multi-morbidity, there is a need to integrate healthcare systems, particularly between primary and secondary healthcare.

The current approaches to surveillance, prevention and treatment of CNCICDs appear to be insufficient to provide for the long-term health needs of this convergence especially in the context of HIV and AIDS era, antiretroviral therapy linked concomitant metabolic complications and HIV/AIDS allied opportunistic infectious diseases. ${ }^{9}$ To address this, the WHO developed the Innovative Care for Chronic Conditions Framework to provide a healthcare systems roadmap that would meet the increasing needs of chronic disease care within this growing multi-morbidity context. This framework incorporates community, patient, healthcare and policy environment perspectives, and has been adopted by different healthcare systems. However, it does not clearly include the infectious diseases within the context of multi-morbidity $^{8}$ and it is then necessary to reorganise healthcare services and systems to tackle this growing public health problem. ${ }^{89}$

The 2016 global diabetes report ${ }^{1}$ emphasises the need to reach better outcomes of diabetes management through an integrated management, especially with NCDs such as cardiovascular diseases as well as tuberculosis and/or HIV/AIDS. This is especially important where the prevalence of these diseases is high. Despite calls for a shift in approach from disease-specific interventions to the integrated delivery model, ${ }^{10}$ healthcare systems in Africa are weak and under-resourced to provide care for the increasing number of patients with multi-morbidities including diabetes, especially compared with high-income settings. ${ }^{11}$

Two types of diabetes commonly identified during adulthood are type 2 diabetes (T2D), that is insulin resistance linked diabetes, and gestational diabetes (GDM), known as a glucose intolerance with onset or first recognition during pregnancy. T2D can be prevented or delayed for women with previous GDM. ${ }^{12-16}$ The established connection between T2D and $\mathrm{GDM}^{9}$ does not determine how GMD is managed. It can either be managed alone in a diabetic clinic or preferably within integrated care at antenatal care (ANC) and postnatal clinics, which is a right approach for increasing multi-morbidity. ${ }^{17} 18$

The treatment pathway for women with GDM is through accessing ANC at the nearest health facility for their pregnancy follow-up and delivery. In contrast, only a small proportion of women with recent GDM return for postpartum oral glucose tolerance test, assessment and management. ${ }^{19-23}$ The main challenge is that GDM women must navigate fragmented health systems for their care and care of their babies and this situation supports calls for integrated health systems and services that are easy for patients to navigate. ${ }^{24}$

Disease-specific or vertical programmes can be used to manage specific diseases and health problems while strengthening fragmented health systems in Africa. ${ }^{25}$ However, disease-specific or stand-alone interventions are criticised for not promoting equity and sustainability of their outcomes, ${ }^{26}$ and therefore integrated programmes to address various NCDs such diabetes in comorbid conditions are recommended. ${ }^{927}$

Integrated care is "combining parts so that they work to form a whole (ie, integration) in order to optimise care and treatment to people where fragmentations in care have led to a negative impact on their care experiences and outcomes". ${ }^{28}$ It describes a range of organisational arrangements with variable nature and intensity and comprises two main concepts: (a) an organisational structure focused on economic benefits (cost-effectiveness), or (b) a way of organising service delivery. ${ }^{29}{ }^{30}$ We conceptualised integration based on dynamic interactions in which formal governance is arranged, responsibilities are shared and resources are pooled, ${ }^{29} 31$ regardless of many other existing integration level models such integrated care typologies used in a recent systematic review that studied the integration of cardiovascular diseases, hypertension and diabetes with HIV services. ${ }^{32}$

The analysis of interactions in health systems enables us to understand the levels of integration. They include partial integration initiatives ranging from (1) the linkage or unstructured interactions, (2) the coordination with a committee to oversee their goal-oriented works but keeping the separated structures, and full integration in which two programmes are merged in their structures (funds, human resources, informational system) and functional elements (strategic planning, resources allocation, interventions delivery). ${ }^{29} 33$

Integrated healthcare systems have advantages such as being associated with more accessibility of care, improved quality and safety of care, healthcare cost reductions and economic benefits for both providers and families. ${ }^{345}$ This integrated management approach including partial and full integration initiatives, will play a key in responding and providing the appropriate healthcare services to the increasing cases of multiple conditions. ${ }^{32}$

We aim to provide a systematic review on integrated management of T2D and GDM within the CNCICDs conditions in Africa. The ultimate goal is to describe the emerging practices and lessons learnt from integrated management of GDM and T2D within comorbidity conditions in Africa and the different research gaps to GDM and T2D integration within management of other non-communicable and infectious chronic diseases.

This systematic review aims to answer the following research questions: (1) What are the existing integrated interventions and services delivery models for managing T2D including GDM within multi-morbidity conditions in Africa? (2) What are the successes and challenges of the 
existing integrated management of T2D including GDM within multi-morbidity conditions?

\section{METHODS}

The Cochrane Handbook and systematic review study protocol $^{36}$ published by the Cochrane Collaboration Methods Groups provides the methodological framework in designing and conducting this systematic review to enable critical appraisal and replication.

\section{Study design}

This systematic review will only include studies of good quality based on the developed inclusion and exclusion criteria.

\section{Patient and public involvement}

There will be no patient and/or public involvement in this study.

\section{Search strategy for the identification of relevant studies}

Our search strategy will use the controlled terms (MeSH: Medical subject heading) and free texts. The following databases will be searched: Cochrane Library, MEDLINE, PubMed and SCOPUS. Other database resources such as the WHO International Clinical Trials Registry Platform, Clinicaltrials.gov, Pan African Clinical Trials Registry (PACTR) and HINARI (Health InterNetwork Access to Research Initiative) will also be searched. Additional search will be conducted in Google scholar. Our research will focus on articles published and grey literature in English and French languages. On the extraction of interesting articles in other languages without any English or French abstracts, we will then include them or get assisted by a researcher who is fluent in those languages. Since there were not many articles regarding our review topic in our preliminary search, there will be no starting time limits in our search but our focus will be limited to all 54 African countries. However, the search will be conducted from the start of each database until the 31 December to include as many relevant studies as possible. Search Strategy will be validated with the assistance of a Librarian.

Our search for articles will be based on the following Population, Intervention, Comparator and Outcome method $^{37}$ (see table 1 below).

\section{Criteria for considering studies for this review}

Studies that have assessed the integrated management of T2D and GDM within multi-morbidity conditions in Africa will be considered, including randomised controlled trials (RCTs), non-RCTs, quasi-randomised controlled trials (QCTs) and observational studies.

Studies will be all kinds of interventions with different targeted participants from all ethnicities, genders, socioeconomic, educational backgrounds and in all countries in Africa who were diagnosed with T2D and GDM as one disease of the multi-morbidity using standard diagnostic criteria will be eligible for inclusion. The patients who had T2D including GDM before and after the occurrence
Table 1 Population, Intervention, Comparator and Outcome description table

\begin{tabular}{|c|c|c|c|}
\hline Population & Intervention & Comparator & Outcomes \\
\hline $\begin{array}{l}\text { Adults } \\
\text { diagnosed } \\
\text { with T2D } \\
\text { and women } \\
\text { diagnosed with } \\
\text { GDM, who } \\
\text { have other } \\
\text { diseases in } \\
\text { public primary } \\
\text { and secondary } \\
\text { healthcare } \\
\text { facilities in } \\
\text { Africa. }\end{array}$ & $\begin{array}{l}\text { Partial or full } \\
\text { Integrated } \\
\text { management } \\
\text { of T2D in } \\
\text { adults and } \\
\text { GDM in } \\
\text { pregnant } \\
\text { women who } \\
\text { have other } \\
\text { diseases in } \\
\text { Africa. }\end{array}$ & $\begin{array}{l}\text { Unintegrated } \\
\text { management } \\
\text { of T2D and } \\
\text { GDM in public } \\
\text { primary and } \\
\text { secondary } \\
\text { healthcare } \\
\text { facilities in } \\
\text { Africa. }\end{array}$ & $\begin{array}{l}\text { Utilisation and } \\
\text { effectiveness } \\
\text { of Integrated } \\
\text { management } \\
\text { of T2D and } \\
\text { GDM in public } \\
\text { primary and } \\
\text { secondary } \\
\text { healthcare } \\
\text { facilities within } \\
\text { multi-morbidity } \\
\text { conditions in } \\
\text { Africa. }\end{array}$ \\
\hline
\end{tabular}

GDM, gestational diabetes; T2D, type 2 diabetes.

of other diseases and the interventions to handle both diseases will be included in this review. Interventions carried out or facilitated by healthcare providers including community health workers in public health facilities will also be included, providing that the focus of the intervention is to treat diseases in which one is diabetes, specifically T2D and GDM. Studies that separately evaluated interventions or assessing vertical programmes of T2D, GDM and other diseases, will be excluded.

\section{Reference lists}

Manual-search by JCM and MW lists of references of included studies, tables of contents of relevant journals and conference abstracts for the relevant material will be conducted. A grey literature search strategy by JCM and MW will be developed to conduct web-based searches to obtain key unpublished sources in our stated search languages.

\section{Selection of studies}

Full copies of articles identified by the search, and considered to meet the inclusion criteria, based on the title and abstract will be obtained for data synthesis. Initially, studies will be screened using predefined inclusion and exclusion criteria. Two reviewers (JCM and MW) will apply the criteria independently to the results of the searches, based first on titles and abstracts only. At least two reviewers (JCM and MW) will proceed independently with the articles selection at the same time based on our described inclusion criteria into two steps: (1) examining the title and abstract, and then, (2) reviewing the full texts. Study authors of eligible articles for which the full text copies are not freely accessible will be contacted to obtain their access and additional information about them will also be requested if required. The inclusion of an article will be made by consensus. In case, the two (JCM and MW) do not reach consensus, the decision from a third person (AR) will be required and reasons for exclusion will be recorded. All studies which initially appear to meet inclusion criteria but on closer inspection 


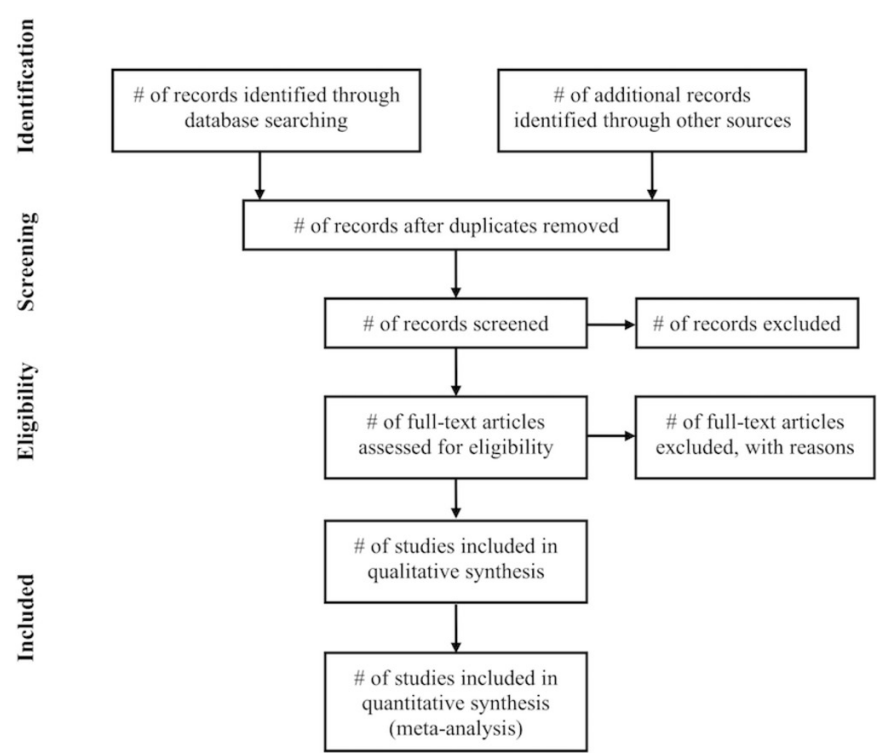

Figure 1 The Preferred Reporting Items for Systematic Reviews and Meta-Analyses flow chart.

do not meet the inclusion criteria will also be detailed in the table of 'characteristics of excluded studies'. The preferred reporting items for systematic review and meta-analysis (PRISMA) flow chart will be produced to facilitate transparency of the process (see figure 1). ${ }^{38} 39$

\section{Types of outcome measures}

Studies reporting at least one of the following outcomes will be included.

\section{Primary outcomes}

Two primary outcomes will be considered: (1) integrated care outcome and (2) cost-effectiveness outcome. For the integrated care outcome, the focus will be on patients screened and/or treated for both T2D and GDM in the course of treatment of other major diseases (eg, HIV, tuberculosis, cardiovascular diseases, etc) in what is known as multi-morbidity conditions. For cost-effectiveness outcome, the focus will be on approach to integrated diagnosis and treatment of T2D and GDM within comorbidity conditions, which simplifies the workload and saves means of depleted health systems in Africa and helps the patients to do not navigate different levels of health systems for their comorbidities that positively impacts the family economies.

\section{Secondary outcomes}

We will also consider early diagnosis through the integrated management of other diseases as this improves clinical outcomes and strengthens health systems for the long-term results of the integrated management of T2D diabetes including GDM within comorbidity conditions.

\section{Quality assessment}

The quality of articles selected will be assessed using the newly developed tool, 'the Cochrane Collaboration Risk of Bias Tool (CCRBT)' and 'Risk Of Bias In Non-randomised Studies - of Interventions (ROBINS-I) ${ }^{40}{ }^{41}$ The
CCRBT is an appropriate quality assessment tool for or RCTs and QCTs. ${ }^{41}{ }^{42}$ Since the quick preliminary search shows that there are few RCTs and QCTs to be included in this study, this single tool will not be enough and the ROBINS-I will be used to assess the quality of non-RCTs and observational studies. ${ }^{41}$ These quality assessment tools encompass all aspects needed to appraise the quality of any studies that will be selected for inclusion.

Two reviewers (JCM and MW) will independently assess the risk of bias in the included studies and cross-checked by a third reviewer (AR).

The following individual quality elements recommended in the modified Cochrane Collaboration tool to assess the risk of bias for RCTs (high, low or unclear) as a judgement for individual elements from six domains (selection, performance, attrition, reporting and other) ${ }^{40}$ will be assessed for RCTs and QCTs. Likewise, the elements recommended in the ROBINS-I to assess risk of bias for non-RCTs and observational studies (low, moderate, serious and critical) as a judgement for individual categories from six domains of bias (bias due to confounding, bias in selection of participants into the study, bias in classification of interventions, bias due to deviations from intended interventions, bias due to missing data, bias in measurement of outcomes and bias in selection of the reported result) ${ }^{41}$ will also be assessed.

Any disagreements over bias between two reviewers will be settled by involving the third review author and each bias in these domains for each study will be separately presented in a table in the final review publication.

\section{Data extraction and management}

As above discussed, the selected citation titles and abstracts will be exported from the search engines to Endnote X8.2 and duplicates will be removed automatically and a search will be conducted manually to check any missed duplicates. Eligible citations will be retrieved after the screening of titles and abstracts and full texts be sought and imported.

JCM and MW will extract data on: study ID, author's name, country, year, type of paper/report, form of publication, study design, comorbidity, description of the intervention (including process, cost-effectiveness and outcomes), context of integrated intervention (ie, PHC, hospitals), details about participants (including number in each group, baseline health information, demographic characteristics), length of intervention and follow-up.

\section{Data analysis and synthesis}

We will first undertake a narrative synthesis to summarise and discuss the findings of the included studies. We will then present findings through primary and secondary outcomes. We will use tabular summary to synthesise individual studies characteristics and results (intervention effects). The data synthesis will be conducted through the measurements of effect for continuous outcomes of the included studies. Studies reporting multiple outcomes and outcome measures will be categories according to 
definitions outline in section types of outcome measure above.

A predetermined order of preference for extracting multiple outcome measures will be used where data is available in several formats. For RCTs, preference will be to extract data that requires the least manipulation by authors or inference by review authors. Raw values (eg, means and SD) rather than calculated effect size will be extracted. For studies reporting both final values and changes from baseline for outcomes, preference will be to extract the former. In the case of cluster-RCTs, the preference will be (i) extract adjusted estimates reported by the study, or (ii) use raw data and inflated the SE data using weighting.

In case of missing data in some eligible studies, efforts will be made to contact corresponding authors to request for clarification of all relevant information. For ongoing studies trial, authors will be contacted for further information and updates.

\section{Statistical analysis and subgroup analysis and investigation of heterogeneity}

Heterogeneity between studies will then be assessed using both $\mathrm{x}^{2}$ and $\mathrm{I}^{2}$ and $\mathrm{Q}$ statistics where appropriate. The $\mathrm{I}^{2}$ statistic estimates the percentage of total variation across studies due to a true difference rather than chance. In general, $\mathrm{I}^{2}$ values greater than $60 \%-70 \%$ indicate the presence of substantial heterogeneity. We will explore sources of heterogeneity by comparing the pooled study estimates between subgroups defined by study-level characteristics. Subgroup analysis will be performed where heterogeneity is statistically significant. Sensitivity analyses will be conducted to determine the potential sources of heterogeneity. Two additional sensitivity analyses will be conducted to: (i) evaluate the effect of excluding studies unable to meet each quality criterion affect the overall estimate, and (ii) evaluate the change in the results if only high-quality studies where included.

In case the identified studies are of substantial heterogeneity and where statistical pooling is impossible, the findings will be summarising in a narrative form by tables and figures to facilitate in effective data presentations. Two reviewers will write the narratives independently and later checked by other reviewers. Decisions on any disagreements will be resolved through discussions and consensus by all reviewers in the team. We will assess the presence of publication bias by using a funnel plot and the Egger test of bias. ${ }^{43}$ Subgroup and sensitivity analyses will be conducted to look at the effects of certain factors on for example: geographic region, age and gender and diabetes type of participating patients.

\section{Reporting of this review}

This protocol complies with the requirements of Preferred Reporting Items for Systematic Reviews and Meta-Analyses Protocol (PRISMA-P), which is included as a online supplementary file 1 . The systematic review results will be reported according to PRISMA. ${ }^{39}$

\section{ETHICS AND DISSEMINATION}

This systematic review will be included in JCM's PhD thesis, a research supervised by Christina Zarowsky (CZ) and Helen Trottier (HT). Its findings will also be disseminated through peer-reviewed publications and conference presentations.

\section{Author affiliations}

'Département de Médecine Sociale et Préventive - Santé Mondiale, École de Santé Publique, Université de Montréal, Montréal, Quebec, Canada

École de santé publique, Université de Montréal, Institut de Recherche en Santé Publique de l'Université de Montréal (IRSPUM), Montreal, Quebec, Canada

${ }^{3}$ Department of Medicine, Faculty of Health Science, University of Cape Town, Chronic Disease Initiative for Africa, Cape Town, South Africa

${ }^{4}$ School of Population and Public Health, University of British Colombia, Vancouver, British Columbia, Canada

${ }^{5}$ Department of Medicine, Faculty of Health Science, University of Cape Town, Integrated Intervention for DIAbetes risks after GestatiOnal diabetes (IINDIAGO), Cape Town, South Africa

${ }^{6}$ Paediatrics and Child Health, University of Witwatersrand, Johannesburg, South Africa

${ }^{7}$ Médecine Sociale et Préventive, École de Santé Publique, Université de Montréal, Montréal, Quebec, Canada

${ }^{8}$ Centre de Recherche du Centre Hospitalier Universitaire Sainte Justine, Montréal, Quebec, Canada

Acknowledgements We would like to thank Sylvie Fontaine, the librarian at the paramedical library, Université de Montréal, Canada, for her valuable contribution to search strategy development and the the reviewers of the manuscript for their constructive inputs. Publication of this protocol was financially supported in part by Institut de Recherche en Santé Publique de l'Université de Montréal (IRSPUM), École de Santé Publique, Université de Montréal.

Contributors JCM and MW designed the study. JCM wrote the first manuscript of the review. JCM, MW, AR, EM, KM, NS, HT, NL and CZ critically revised the review. All authors read and approved the final manuscript. JCM is an MHA, MSc, MA, MPhil and $\mathrm{PhD}$ candidate in Public Health-Global Health. MW is an MD, MPH and PhD candidate in Public Health. AR is an MPH and PhD in Healthcare and EpidemiologyGlobal Health. EM is an MBBS and Fellow of West African College of Physicians (FWACP) in Internal Medicine-Endocrinology. KM is a BA, HDE, PGDip. in Health Promotion and PhD in Public Health. NS is a BSc, BSc (Hon.) and PhD in Health Sciences. HT is an MSc and PhD in Epidemiology. NL is an MBChB, MD and Fellow of College Physicians of South Africa (FCP-SA)-Endocrinology/Diabetology. CZ is an $\mathrm{MD}, \mathrm{MPH}$ and $\mathrm{PhD}$ in Medical Anthropology.

Funding The authors have not declared a specific grant for this research from any funding agency in the public, commercial or not-for-profit sectors.

Competing interests None declared.

Patient consent for publication Not required.

Ethics approval Given that this is a protocol for a systematic review only using the published data, there is no ethics approval required for this study.

Provenance and peer review Not commissioned; externally peer reviewed.

Open access This is an open access article distributed in accordance with the Creative Commons Attribution Non Commercial (CC BY-NC 4.0) license, which permits others to distribute, remix, adapt, build upon this work non-commercially, and license their derivative works on different terms, provided the original work is properly cited, appropriate credit is given, any changes made indicated, and the use is non-commercial. See: http://creativecommons.org/licenses/by-nc/4.0/.

\section{REFERENCES}

1. WHO. Global report on diabetes 2016. 2018 http://www.who.int/ diabetes/global-report/en/

2. Ogurtsova K, da Rocha Fernandes JD, Huang Y, et al. IDF Diabetes Atlas: global estimates for the prevalence of diabetes for 2015 and 2040. Diabetes Res Clin Pract 2017;128:40-50.

3. Day C. Reflections from IDF-WDC 2015. British Journal of Diabetes 20162016;16:37-8. 
4. Aguiree FB, Cho A, Dahlquist NH, et al. IDF Diabetes Atlas. 2013 http://dro.deakin.edu.au/view/DU:30060687

5. Dalal S, Beunza JJ, Volmink J, et al. Non-communicable diseases in sub-Saharan Africa: what we know now. Int J Epidemiol 2011;40:885-901.

6. McKeown RE. The Epidemiologic Transition: Changing Patterns of Mortality and Population Dynamics. Am J Lifestyle Med 2009;3(1 Suppl):19S-26.

7. Howard AA, EI-Sadr WM. Integration of tuberculosis and HIV services in sub-Saharan Africa: lessons learned. Clin Infect Dis 2010;50(Suppl 3):S238-44.

8. Oni T, McGrath N, BeLue R, et al. Chronic diseases and multimorbidity--a conceptual modification to the WHO ICCC model for countries in health transition. BMC Public Health 2014;14:575.

9. Levitt NS, Steyn K, Dave J, et al. Chronic noncommunicable diseases and HIV-AIDS on a collision course: relevance for health care delivery, particularly in low-resource settings--insights from South Africa. Am J Clin Nutr 2011;94:1690S-6.

10. Fleury MJ. Integrated service networks: the Quebec case. Health Serv Manage Res 2006;19:153-65.

11. Iwelunmor J, Blackstone S, Veira D, et al. Toward the sustainability of health interventions implemented in sub-Saharan Africa: a systematic review and conceptual framework. Implement Sci 2016:11:43.

12. Ruchat SM, Mottola MF. The important role of physical activity in the prevention and management of gestational diabetes mellitus. Diabetes Metab Res Rev 2013;29:334-46.

13. American Diabetes Association and National Institute of Diabetes, Digestive and Kidney Diseases. The prevention or delay of type 2 diabetes. Diabetes Care 2002;25:742-9.

14. Tuomilehto J, Lindström J, Eriksson JG, et al. Prevention of type 2 diabetes mellitus by changes in lifestyle among subjects with impaired glucose tolerance. N Engl J Med 2001;344:1343-50.

15. Alberti KG, Zimmet PZ. Definition, diagnosis and classification of diabetes mellitus and its complications. Part 1: diagnosis and classification of diabetes mellitus provisional report of a WHO consultation. Diabet Med 1998;15:539-53.

16. Buchanan TA, Xiang A, Kjos SL, et al. What is gestational diabetes? Diabetes Care 2007;30(Suppl 2):S105-11.

17. Struckmann V, Leijten FRM, van Ginneken E, et al. SELFIE consortium. Relevant models and elements of integrated care for multi-morbidity: Results of a scoping review. Health Policy 2018;122:23-35.

18. Fealy SM, Taylor RM, Foureur M, et al. Weighing as a stand-alone intervention does not reduce excessive gestational weight gain compared to routine antenatal care: a systematic review and metaanalysis of randomised controlled trials. BMC Pregnancy Childbirth 2017;17:36.

19. Ben-Haroush A, Yogev Y, Hod M. Epidemiology of gestational diabetes mellitus and its association with Type 2 diabetes. Diabet Med 2004;21:103-13.

20. Hartling L, Dryden DM, Guthrie A, et al. Screening and diagnosing gestational diabetes mellitus. Evid Rep Technol Assess 2012:1-327.

21. Hartling L, Dryden DM, Guthrie A, et al. Benefits and harms of treating gestational diabetes mellitus: a systematic review and metaanalysis for the U.S. Preventive Services Task Force and the National Institutes of Health Office of Medical Applications of Research. Ann Intern Med 2013;159:123-9.

22. Nicklas JM, Zera CA, Seely EW, et al. Identifying postpartum intervention approaches to prevent type 2 diabetes in women with a history of gestational diabetes. BMC Pregnancy Childbirth 2011;11:23.
23. Bennett WL, Ennen CS, Carrese JA, et al. Barriers to and facilitators of postpartum follow-up care in women with recent gestational diabetes mellitus: a qualitative study. $J$ Womens Health 2011;20:239-45.

24. Linenkugel N. Integrated health networks are not created equal. Front Health Serv Manage 2001;17:41-4.

25. Van Damme W. How can disease control programs contribute to health systems strengthening in sub-Saharan Africa? Which health systems for disease control, 2011.

26. Atun RA. When do vertical (stand alone) programmes have a place in health systems? 2008.

27. Haregu TN, Setswe G, Elliott J, et al. Integration of HIV/AIDS and noncommunicable diseases in developing countries: rationale, policies and models. Int $J$ Healthc 2015;1:21.

28. Goodwin N. Understanding integrated care: a complex process, a fundamental principle. Int J Integr Care 2013;13.

29. Shigayeva A, Atun R, McKee M, et al. Health systems, communicable diseases and integration. Health Policy Plan 2010;25(Suppl 1):i4-20.

30. Tudor Car L, van-Velthoven MH, Brusamento S, et al. Integrating prevention of mother-to-child HIV transmission (PMTCT) programmes with other health services for preventing HIV infection and improving HIV outcomes in developing countries. Cochrane Database Syst Rev 2011;6:Cd008741.

31. Messeri P, Kim S, Whetten K. Measuring HIV Services Integration Activities. J HIV AIDS Soc Serv 2003;2:19-44.

32. Haldane V, Legido-Quigley H, Chuah FLH, et al. Integrating cardiovascular diseases, hypertension, and diabetes with HIV services: a systematic review. AIDS Care 2018;30:103-15.

33. Atun R, de Jongh T, Secci F, et al. Integration of targeted health interventions into health systems: a conceptual framework for analysis. Health Policy Plan 2010;25:104-11.

34. Coddington DC, Moore KD, Fischer EA. Costs and benefits of integrated healthcare systems. Healthc Financ Manage 1994:48:20-4.

35. Henke KD. [What are the economic advantages of Integrated Health Care?]. Clin Res Cardiol 2006;95(Suppl 2):li11-12.

36. Higgins JP, Altman DG. Assessing risk of bias in included studies. Cochrane handbook for systematic reviews of interventions: cochrane book series, 2008:187-241.

37. Counsell C. Formulating questions and locating primary studies for inclusion in systematic reviews. Ann Intern Med 1997;127:380-7.

38. Werfalli M, Musekiwa A, Engel ME, et al. The prevalence of type 2 diabetes mellitus among older people in Africa: a systematic review study protocol. BMJ Open 2014;4:e004747.

39. Moher D, Liberati A, Tetzlaff J, et al. Preferred reporting items for systematic reviews and meta-analyses: the PRISMA statement. PLOS Med 2009;6:e1000097.

40. Higgins JP, Altman DG, Gøtzsche PC, et al. The Cochrane Collaboration's tool for assessing risk of bias in randomised trials. BMJ 2011;343:d5928.

41. Sterne JA, Hernán MA, Reeves BC, et al. ROBINS-I: a tool for assessing risk of bias in non-randomised studies of interventions. BMJ 2016;355:i4919.

42. Armijo-Olivo S, Stiles CR, Hagen NA, et al. Assessment of study quality for systematic reviews: a comparison of the Cochrane Collaboration Risk of Bias Tool and the Effective Public Health Practice Project Quality Assessment Tool: methodological research. J Eval Clin Pract 2012;18:12-18.

43. Ukoumunne OC, Gulliford MC, Chinn S, et al. Methods for evaluating area-wide and organisation-based interventions in health and health care: a systematic review. Health Technol Assess 1999;3:iii-92. 